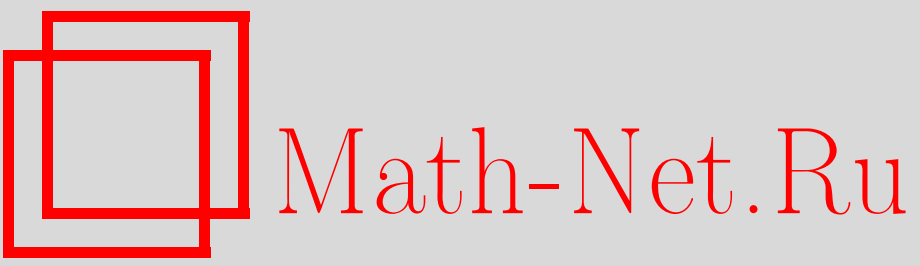

А. С. Шведов, О нечетких множествах типа 2 и нечетких системах типа 2, Итоги науки и техн. Сер. Соврем. мат. и ее прил. Темат. обз., 2019, том 165, $114-$ 122

DOI: https://doi.org/10.36535/0233-6723-2019-165-114-122

Использование Общероссийского математического портала Math-Net.Ru подразумевает, что вы прочитали и согласны с пользовательским соглашением http://www . mathnet.ru/rus/agreement

Параметры загрузки:

IP : 18.234 .197 .8

26 апреля 2023 г., $16: 58: 35$ 


\title{
О НЕЧЕТКИХ МНОЖКСТВАХ ТИПА 2 И НЕЧЕТКИХ СИСТЕМАХ ТИПА 2
}

\author{
(c) 2019 г. $\quad$ А. С. ШВЕДОВ
}

\begin{abstract}
АннотАция. Одним из преимуществ систем, основанных на нечеткой логике (нечетких систем), является возможность мягкого переключения от одних значений входных параметров системы к другим, когда делаются разные выводы для различных областей этих значений. Нечеткое множество типа 2-это прямое обобщение обычного нечеткого множества. В настоящей работе дается обзор некоторых разделов теории нечетких множеств типа 2 и теории нечетких систем типа 2. Рассматриваются операции над нечеткими множествами типа 2 , нечеткие отношения типа 2 , центроид нечеткого множества типа 2. Дается описание функциональных нечетких систем типа 2 и реляционных нечетких систем типа 2.
\end{abstract}

Ключевые слова: нечеткое множество типа 2, нечеткое отношение типа 2, функциональная нечеткая система, реляционная нечеткая система.

\section{ON TYPE-2 FUZZY SETS AND TYPE-2 FUZZY SYSTEMS}

\section{(c) 2019 A. S. SHVEDOV}

\begin{abstract}
One of the advantages of systems based on fuzzy logic (fuzzy systems) is the possibility of a soft switch from one set of values of input parameters of the system to another, when different conclusions are drawn for different sets of these values. A fuzzy set of type 2 is a direct generalization of an ordinary fuzzy set. In this paper, we review some branches of the theory of type- 2 fuzzy sets and the theory of type- 2 fuzzy systems. We discuss operations on type- 2 fuzzy sets, type- 2 fuzzy relations, and the centroids of type-2 fuzzy sets and describe type- 2 functional fuzzy systems and type- 2 relational fuzzy systems.
\end{abstract}

Keywords and phrases: fuzzy set of type 2, fuzzy relation of type 2, functional fuzzy system, relational fuzzy system.

AMS Subject Classification: 93C42, 94D05

\begin{abstract}
Введение. В классической теории множеств для любого подмножества некоторого множества (которое будем называть универсальным множеством) степень принадлежности элемента универсального множества данному подмножеству может равняться либо 0 (элемент подмножеству не принадлежит), либо 1 (элемент подмножеству принадлежит). В теории нечетких множеств степень принадлежности элемента универсального множества нечеткому подмножеству (нечеткому множеству) может быть любым действительным числом, лежащим между 0 и 1 . В теории нечетких множеств типа 2 принимается, что и сама эта степень принадлежности не определяется для каждого элемента универсального множества однозначно, допускается некоторая размытость. Точнее, степень принадлежности является нечетким числом, носитель которого принадлежит отрезку $[0,1]$.
\end{abstract}


Теория нечетких множеств имеет большое и теоретическое, и практическое значение при математическом моделировании неопределенности, по этому научному направлению имеется обширная литература (см, например, книги $[1,3]$ и библиографию в них). Данная теория позволяет передать другой вид неопределенности, нежели теория вероятностей. В теории вероятностей каждой группе значений неизвестной величины приписывается некоторая вероятность. В теории нечетких множеств сами значения определяются неоднозначно. Теория нечетких множеств типа 2 и нечетких систем типа 2 - это научное направление, которое находится в состоянии интенсивного развития и имеет большое практическое значение (см., например, книги $[7,20])$.

Нечеткие множества типа 2 введены в [28]. Теория этих множеств получила дальнейшее развитие в $[21,22]$. Обзоры теории и ее приложений в различных прикладных областях приведены, например, в $[15,18]$. Из тематически более узких обзоров можно назвать $[8,13,23,25]$. Различные результаты, относящиеся к теории нечетких систем типа 2, представлены, в частности, в $[14,16,19,26,30]$, а также во многих других работах.

Под нечеткими системами многие понимают нечеткие системы управления. Такие системы рассматриваются, например, в $[11,12,17,24,27,29]$. (Классический пример нечеткой системы управления следующий. Если препятствие близко и скорость большая, то тормозить надо резко; если препятствие далеко или скорость малая, то тормозить надо плавно. Здесь используются два нечетких правила и шесть нечетких множеств, точнее, следующие три связки нечетких множеств: близкое препятствие, далекое препятствие; малая скорость, большая скорость; плавное торможение, резкое торможение. Например, степень принадлежности скорости 40 км/час к нечеткому множеству больших скоростей в зависимости от задачи может быть принята равной 0,3 , но может быть принята и равной 0,8 . Степень принадлежности скорости 60 км/час к нечеткому множеству больших скоростей для этих задач может быть, соответственно, 0,5 и 0,9 . Таким образом учитывается неоднозначность понятия (значения) «большая скорость». Подобные связки называются лингвистическими переменными. Разумеется, в связке может быть и больше двух нечетких множеств.) Однако термин «нечеткие системы» все же более широк, чем «нечеткие системы управления». Например, в $[9,10]$ представлен программный комплекс, предназначенный для исследования рынка акций и выработки торговых стратегий, в основе которого лежат нечеткие системы, но управления в модель не включаются. Подобные нечеткие системы эконометрической направленности рассматриваются и другими авторами. Нечеткие системы называют также системами, основанными на нечеткой логике. Одним из преимуществ такого подхода является возможность мягкого переключения. Так, в рассмотренном примере производится мягкое переключение от ситуации с близким препятствием к ситуации с далеким препятствием.

Если в нечетких правилах используются нечеткие множества типа 2, то говорят о нечетких системах типа 2. Такая модификация позволяет добиться лучших результатов в ряде задач. Нечеткие системы типа 2, как и обычные нечеткие системы, делятся на функциональные и реляционные. Обзоров по теории нечетких систем типа 2 на русском языке немного; укажем, например, $[4$, гл. 5] и [2]. Однако ни в одной из этих двух работ не рассматриваются, например, функциональные нечеткие системы типа 2. Настоящий обзор в определенной степени заполняет имеющийся пробел.

Широкое использование нечетких систем типа 1 в различных прикладных областях во многом связано с тем, что такие системы являются универсальными аппроксиматорами (см., например, обзор [6]). Зависимость между входными и выходными переменными системы может иметь нелинейный характер, заранее не известный. Нечеткие системы позволяют дать правильную аппроксимацию для широкого класса таких зависимостей. Разумеется, универсальными аппроксиматорами являются и нечеткие системы типа 2 , поскольку это более широкий класс систем, чем нечеткие системы типа 1. Развитие теории нечетких систем типа 2 и применение этой теории является перспективным научным направлением.

Настоящая работа имеет следующую структуру. В разделе 1 приведены необходимые определения, введены операции над нечеткими множествами типа 2. В разделе 2 описан центроид нечеткого множества типа 2, рассмотрены функциональные нечеткие системы типа 2. В разделе 3 приведены определения, относящиеся к нечетким отношениям типа 2, рассмотрены расширенные 
$t$-норма и $t$-конорма, а также реляционные нечеткие системы типа 2 . Определения и исторические ссылки, относящиеся к обычным нечетким множествам, в настоящей работе не обсуждаются; их можно найти, например, в обзоре [5].

1. Нечеткие множества типа 2. Пусть $X$ - некоторое универсальное множество. Для обычного нечеткого множества $A$ степень принадлежности каждого элемента $x \in X$ этому нечеткому множеству является действительным числом $\mu_{A}(x)$, принадлежащим отрезку $[0,1]$.

Однако во многих приложениях остается неясным, как наилучшим образом определить степень принадлежности каждого элемента $x$ нечеткому множеству $A$. Поэтому возникла идея, что сама степень принадлежности может быть нечетким множеством, носитель которого принадлежит отрезку $[0,1]$. Оказывается, что такой подход позволяет получить лучшие результаты в ряде прикладных задач.

Рассмотрим функцию $\mu: X \times[0,1] \rightarrow[0,1]$.

Определение 1. График функции $\mu$, т.е. множество пар

$$
((x, w), \mu(x, w)), \quad x \in X, w \in[0,1],
$$

называется нечетким множеством типа 2 (НMT2).

Обычные нечеткие множества будем называть нечеткими множествами типа 1 (HMT1).

Замечание. Можно было бы рассматривать HMT2 как HMT1 с универсальным множеством $X \times[0,1]$. Однако такой подход не является удобным, например, при введении операций объединения, пересечения, дополнения для НМТ2. Кроме того, по смыслу рассматриваемых прикладных задач универсальным множеством является $X$, а не $X \times[0,1]$. Функцию $\mu$ для НМТ2 $A$ обозначаем $\mu_{A}$.

Пусть $A-\mathrm{HMT2;} \mathrm{для} \mathrm{каждого} x \in X$ рассмотрим множество

$$
J_{x}=\left\{w \in[0,1]: \mu_{A}(x, w)>0\right\} .
$$

В приводимых ниже определениях 2-7 $A$-это НMT2.

Определение 2. Первичной функиией принадлежности $A$ называется многозначная функция, которая каждому элементу $x \in X$ ставит в соответствие множество $J_{x}$.

Определение 3. Нижней функиией принадлежности $A$ называется функция $\underline{\mu}_{A}: X \rightarrow[0,1]$, определяемая соотношением

$$
\underline{\mu}_{A}(x)=\inf J_{x}, \quad x \in X .
$$

Определение 4. Верхней функиией принадлежности $A$ называется функция $\bar{\mu}_{A}: X \rightarrow[0,1]$, определяемая соотношением

$$
\bar{\mu}_{A}(x)=\sup J_{x}, \quad x \in X .
$$

Определение 5. Если при каждом $x \in X$ существует единственный элемент $w_{x} \in[0,1]$, для которого что $\mu_{A}\left(x, w_{x}\right)=1$, то функция, которая элементу $x$ ставит в соответствие элемент $w_{x}$, называется главной функцией принадлежсности $A$.

Определение 6. При фиксированном $x \in X$ функция $\mu_{A}(x, w)$ как функция аргумента $w$ называется вторичной функиией принадлежности $A$.

Определение 7. Изображением неопределенности $A$ называется множество

$$
F O U(A)=\left\{(x, w): x \in X, w \in J_{x}\right\} .
$$

Определение 8. НМТ2 $A$ называется интервалъным нечетким множеством типа 2 (ИНМТ2), если $\mu_{A}(x, w)=1$ при всех $x \in X, w \in J_{x}$.

Определение 9. НMT1 $C$ с универсальным множеством $X$ называется встроенным в НМТ2 $A$, если

при всех $x \in X$.

$$
\underline{\mu}_{A}(x) \leqslant \mu_{C}(x) \leqslant \bar{\mu}_{A}(x)
$$


Замечание. Можно рассматривать и нечеткие множества типа 3 , когда для любых $x \in X$, $w \in[0,1]$ значение $\mu(x, w)$ является не действительным числом, а НМТ1 с носителем, принадлежащим отрезку $[0,1]$, а также нечеткие множества более высоких типов. Однако практическое значение таких математических конструкций, видимо, является несопоставимо меньшим, чем практическое значение нечетких множеств типов 1 и 2.

При работе с НMT2 используется обычное для НМТ1 обозначение:

$$
A=\int_{x \in X} \int_{w \in[0,1]} \frac{\mu_{A}(x, w)}{(x, w)},
$$

причем знак $\int$ часто используется вместо знака $\sum$ и в тех случаях, когда каждое из множеств $X$, $J_{x}$ содержит лишь конечное число элементов. Иногда последнюю формулу удобнее записывать в виде

$$
A=\int_{x \in X}\left(\int_{w \in[0,1]} \frac{\mu_{A}(x, w)}{w}\right) / x
$$

подчеркивая, что каждому $x$ ставится в соответствие некоторое HMT1, носитель которого принадлежит отрезку $[0,1]$.

Если при $0<\eta \leqslant 1$ рассмотреть $\eta$-срезы НMT2 A:

$$
A_{\eta}=\left\{(x, w): \mu_{A}(x, w) \geqslant \eta\right\},
$$

Tо

$$
\mu_{A}(x, w)=\sup _{\eta \in(0,1]} \eta \cdot I_{A_{\eta}}(x, w)
$$

при всех $x \in X, w \in[0,1]$. Здесь $I_{A_{\eta}}$ - индикатор множества $A_{\eta}$. Доказательство последней формулы ничем не отличается от доказательства аналогичной формулы для HMT1.

Пусть $A$ и $B-$ HМТ2 с одним и тем же универсальным множеством $X$.

Определение 10. Объединением $A$ и $B$ называется НМТ2

$$
A \cup B=\int_{x \in X}\left(\int_{w \in[0,1]} \frac{\sup _{u \in[0,1], v \in[0,1]: u \vee v=w} \mu_{A}(x, u) \wedge \mu_{B}(x, v)}{w}\right) / x .
$$

Здесь $\wedge$ означает минимум, $\vee-$ максимум.

Определение 11. Пересечением $A$ и $B$ называется НМТ2

$$
A \cap B=\int_{x \in X}\left(\int_{w \in[0,1]} \frac{\sup _{u \in[0,1], v \in[0,1]: u \wedge v=w} \mu_{A}(x, u) \wedge \mu_{B}(x, v)}{w}\right) / x .
$$

Определение 12. Дополнением A называется НМТ2

$$
\bar{A}=\int_{x \in X}\left(\int_{w \in[0,1]} \frac{\mu_{A}(x, w)}{1-w}\right) / x .
$$

2. Центроид нечеткого множества типа 2 и функциональные нечеткие системы. Пусть универсальное множество $X$-это конечное множество, элементами которого являются действительные числа, $X=\left\{x_{1}, \ldots, x_{m}\right\}$.

Напомним, что если $A$-это НMT1, то центроидом (центром тяжести) $A$ называется действительное число

$$
\left(\sum_{i=1}^{m} x_{i} \mu_{A}\left(x_{i}\right)\right) /\left(\sum_{i=1}^{m} \mu_{A}\left(x_{i}\right)\right) .
$$


Пусть $A$-это НМТ2 и каждое из множеств $J_{x_{i}}, i=1, \ldots, m$, содержит лишь конечное число элементов. Задача состоит в том, чтобы построить HMT1, которое могло бы рассматриваться как центроид $A$.

Пусть носителем искомого НМТ1 является множество всех действительных чисел вида

$$
\left(\sum_{i=1}^{m} x_{i} w_{i}\right) /\left(\sum_{i=1}^{m} w_{i}\right)
$$

где $w_{1} \in J_{x_{1}}, \ldots, w_{m} \in J_{x_{m}}$. Это объединение центроидов всех встроенных HMT1. Если множество $J_{x_{i}}$ содержит $n_{i}$ элементов, то всего чисел вида $(1)$ может быть $\prod_{i=1}^{m} n_{i}$. Но некоторые из этих чисел могут совпадать.

Для действительного числа вида (1) степень принадлежности центроиду $A$ принимается равной

$$
\mu_{A}\left(x_{1}, w_{1}\right) \wedge \cdots \wedge \mu_{A}\left(x_{m}, w_{m}\right) .
$$

Интересно (см. [16]), что в данном случае вместо минимума нельзя использовать произведение. Если какой-то элемент носителя допускает несколько различных представлений вида (1), то в качестве его степени принадлежности HMT1 в соответствии с принципом расширения нужно брать максимальное из чисел (2).

Построенный таким образом центроид НMT2 $A$ обозначается $C_{A}$ и является, как уже было сказано, НМТ1.

Если $A$ - это ИНМТ2, то каждое из чисел (2) равняется 1.

Перейдем к случаю, когда каждое из множеств $J_{x_{i}}, i=1, \ldots, m$, является отрезком действительной прямой (принадлежащим отрезку $[0,1]) ; A$ - ИНМТ2. Задача вновь состоит в том, чтобы построить НMT1, которое могло бы рассматриваться как центроид $A$.

Пусть носителем искомого НМТ1 является множество действительных чисел вида (1), где $w_{1} \in J_{x_{1}}, \ldots, w_{m} \in J_{x_{m}}$. Нетрудно увидеть, что построенный таким образом носитель является отрезком действительной прямой, который мы обозначим через $\left[y_{l}, y_{r}\right]$.

Действительно, если

$$
y^{\prime}=\left(\sum_{i=1}^{m} x_{i} w_{i}^{\prime}\right) /\left(\sum_{i=1}^{m} w_{i}^{\prime}\right), \quad y^{\prime \prime}=\left(\sum_{i=1}^{m} x_{i} w_{i}^{\prime \prime}\right) /\left(\sum_{i=1}^{m} w_{i}^{\prime \prime}\right),
$$

то для любого $y \in\left[y^{\prime}, y^{\prime \prime}\right]$ на отрезке, соединяющем точки $\left(w_{1}^{\prime}, \ldots, w_{m}^{\prime}\right)$ и $\left(w_{1}^{\prime \prime}, \ldots, w_{m}^{\prime \prime}\right)$, найдется точка $\left(w_{1}, \ldots, w_{m}\right)$, для которой значение дроби (1) равно $y$, поскольку функция (1) как функция аргумента $\left(w_{1}, \ldots, w_{m}\right)$ непрерывна на этом отрезке. Ограниченность функции $(1)$ как функции аргумента $\left(w_{1}, \ldots, w_{m}\right)$ при фиксированных $x_{1}, \ldots, x_{m}$ очевидна. Очевидна и замкнутость множества всех значений функции $(1)$ при $\left(w_{1}, \ldots, w_{m}\right) \in J_{x_{1}} \times \cdots \times J_{x_{m}}$. Отдельно следует рассмотреть случай, когда $0 \in J_{x_{1}}, \ldots, 0 \in J_{x_{m}}$. В этом случае, если каждый из отрезков $J_{x_{1}}, \ldots, J_{x_{m}}$ имеет ненулевую длину, то $\left[y_{l}, y_{r}\right]=\left[\min _{1 \leqslant i \leqslant m} x_{i}, \max _{1 \leqslant i \leqslant m} x_{i}\right]$. В точке $(0, \ldots, 0)$ функция (1) при этом не определена.

Чтобы завершить построение центроида $C_{A}$, для каждого $y \in\left[y_{l}, y_{r}\right]$ положим $\mu_{C_{A}}(y)=1$.

Функциональные нечеткие системы называются также нечеткими системами Такаги-Сугено. Рассмотрим систему, для которой входом являются действительные числа $x_{1}^{0}, \ldots, x_{n}^{0}$, выходомдействительное число $y^{0}$. Пусть $x_{1}^{0} \in X_{1}, \ldots, x_{n}^{0} \in X_{n}, y^{0} \in Y$, где $X_{1}, \ldots, X_{n}, Y$ - некоторые подмножества множества действительных чисел $\mathbb{R}$.

База состоит из $k$ нечетких правил следующего вида:

$$
\text { ЕСЛИ }\left(x_{1}=A_{j 1}\right) \text { И ... И }\left(x_{n}=A_{j n}\right) \text {, ТО } y=f_{j}\left(x_{1}, \ldots, x_{n}\right),
$$

где $j=1, \ldots, k$. При каждом $j$ считается, что $A_{j p}$-ИНМТ2 с универсальным множеством $X_{p}$, $p=1, \ldots, n, f_{j}: X_{1} \times \cdots \times X_{n} \rightarrow \mathbb{R}, j=1, \ldots, k$, - некоторые функции.

Суть нечеткой системы состоит в том, что для различных областей значений входных параметров $\left(x_{1}^{0}, \ldots, x_{n}^{0}\right)$ требуется делать различные выводы (использовать различные формы зависимости выходных параметров от входных) и обеспечивать при этом мягкое переключение. 
Если степень активации нечеткого правила $j$ при данном наборе входных параметров большая, то вклад зависимости $f_{j}$ в окончательную общую зависимость также большой, и наоборот, при малой степени активации нечеткого правила вклад малый.

Описание нечетких систем Такаги-Сугено для случая, когда $A_{j p}, p=1, \ldots, n,-\mathrm{HMT} 1$, можно найти, например, в [3].

Замечание. Наиболее важен случай, когда функции $f_{1}, \ldots, f_{k}$ являются линейными:

$$
f_{j}\left(x_{1}, \ldots, x_{n}\right)=a_{j 0}+a_{j 1} x_{1}+\cdots+a_{j n} x_{n}
$$

где $a_{j 0}, a_{j 1}, \ldots, a_{j n}$ - действительные числа. Теоретически могут рассматриваться и модели, для которых $a_{j 0}, a_{j 1}, \ldots, a_{j n}-\mathrm{HMT1}$, но на практике такие модели, видимо, пока не используются.

Пусть $\underline{\mu}_{A_{j 1}}, \ldots, \underline{\mu}_{A_{j n}}$ и $\bar{\mu}_{A_{j 1}}, \ldots, \bar{\mu}_{A_{j n}}$ - соответственно нижние и верхние функции принадлежности ИНМТ2 $A_{j 1}, \ldots, A_{j n} ; j=1, \ldots, k$.

При каждом $j, 1 \leqslant j \leqslant k$, рассмотрим отрезки $\left[\underline{\tau}_{j}, \bar{\tau}_{j}\right]$, где

или

$$
\underline{\tau}_{j}=\min \left(\underline{\mu}_{A_{j 1}}\left(x_{1}^{0}\right), \ldots, \underline{\mu}_{A_{j n}}\left(x_{n}^{0}\right)\right), \quad \bar{\tau}_{j}=\min \left(\bar{\mu}_{A_{j 1}}\left(x_{1}^{0}\right), \ldots, \bar{\mu}_{A_{j n}}\left(x_{n}^{0}\right)\right),
$$

$$
\underline{\tau}_{j}=\underline{\mu}_{A_{j 1}}\left(x_{1}^{0}\right) \cdots \underline{\mu}_{A_{j n}}\left(x_{n}^{0}\right), \quad \bar{\tau}_{j}=\bar{\mu}_{A_{j 1}}\left(x_{1}^{0}\right) \cdots \bar{\mu}_{A_{j n}}\left(x_{n}^{0}\right) .
$$

Могут использоваться и другие $t$-нормы, кроме минимума и произведения. (Определения $t$-нормы и $t$-конормы см., например, в [4].) Интервальное НMT1 с носителем $\left[\underline{\tau}_{j}, \bar{\tau}_{j}\right]$ называется степенъю активачии $j$-го нечеткого правила.

Для определения выхода нечеткой системы степень активации каждого нечеткого правила должна быть увязана с выходным значением для этого нечеткого правила. Рассматривается отрезок $\left[y_{l}, y_{r}\right]$, состоящий из всех точек вида

$$
\left(\sum_{j=1}^{k} \tau_{j} f_{j}\left(x_{1}^{0}, \ldots, x_{n}^{0}\right)\right) /\left(\sum_{j=1}^{k} \tau_{j}\right),
$$

где $\tau_{j} \in\left[\underline{\tau}_{j}, \bar{\tau}_{j}\right], j=1, \ldots, k$. Обоснование того, что данное множество точек является отрезком, аналогично приведенному выше. Таким образом,

$$
y_{l}=\min _{\substack{\tau_{j} \in\left[\underline{\tau}_{j}, \bar{\tau}_{j}\right] \\ j=1, \ldots, k}} \frac{\sum_{j=1}^{k} \tau_{j} f_{j}\left(x_{1}^{0}, \ldots, x_{n}^{0}\right)}{\sum_{j=1}^{k} \tau_{j}}, \quad y_{r}=\max _{\substack{\tau_{j} \in\left[\underline{\tau}_{j}, \bar{\tau}_{j}\right] \\ j=1, \ldots, k}} \frac{\sum_{j=1}^{k} \tau_{j} f_{j}\left(x_{1}^{0}, \ldots, x_{n}^{0}\right)}{\sum_{j=1}^{k} \tau_{j}} .
$$

Далее, $y^{0}=\left(y_{l}+y_{r}\right) / 2$.

Представленный способ нахождения $y^{0}$ является одним из наиболее распространенных. Но можно использовать и более простой способ, когда $y^{0}$ рассчитывается по формуле (3), в которой $\tau_{j}=\left(\underline{\tau}_{j}+\bar{\tau}_{j}\right) / 2$.

3. Нечеткие отношения типа 2 и реляционные нечеткие системы. Пусть $X$ и $Y$-два множества, которые используются в качестве универсальных множеств.

Определение 13. Нечетким отношением типа 2 между множествами $X$ и $Y$ называется НMT2 с универсальным множеством $X \times Y$.

Пусть $D_{1}, \ldots, D_{n}-\mathrm{HMT1}$, универсальным множеством для каждого из которых является отрезок $[0,1] ; \mu_{D_{1}}, \ldots, \mu_{D_{n}}-$ функции принадлежности этих нечетких множеств.

Пусть $\Delta$-некоторая $t$-норма, $\nabla$-некоторая $t$-конорма. Как известно, для любых чисел $a_{1}, \ldots, a_{n}$, принадлежащих отрезку $[0,1], a_{1} \Delta \ldots \Delta a_{n}$ и $a_{1} \nabla \ldots \nabla a_{n}$-также некоторые числа, принадлежащие отрезку $[0,1]$. Распространенными примерами $t$-норм являются операции произведения и взятия минимума; примером $t$-конормы является операция взятия максимума. 
В теории нечетких систем типа 2 используются операции расширенной $t$-нормы $*$ и расширенной $t$-конормы $\stackrel{\nabla}{*}$. Эти операции применяются к функциям принадлежности $\mu_{D_{1}}, \ldots, \mu_{D_{n}}$, и результатом является также некоторая функция принадлежности, носитель которой принадлежит отрезку $[0,1]$. Пусть $\bar{\Delta}$ - некоторая $t$-норма, которая может совпадать или не совпадать с $t$-нормой $\Delta$.

Определение 14. Результатом применения расширенной $t$-нормъ $*$ к функциям принадлежности $\mu_{D_{1}}, \ldots, \mu_{D_{n}}$ является функция принадлежности

$$
\left(\mu_{D_{1}} * \ldots * \mu_{D_{n}}\right)(w)=\sup _{\substack{u_{1} \in[0,1], \ldots, u_{n} \in[0,1]: \\ u_{1} \Delta \ldots \Delta u_{n}=w}} \mu_{D_{1}}\left(u_{1}\right) \bar{\Delta} \ldots \bar{\Delta} \mu_{D_{n}}\left(u_{n}\right),
$$

где $w \in[0,1]$.

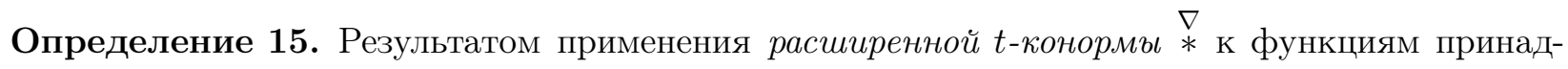
лежности $\mu_{D_{1}}, \ldots, \mu_{D_{n}}$ является функция принадлежности

$$
\left(\mu_{D_{1}} * \ldots * \mu_{D_{n}}\right)(w)=\sup _{\substack{u_{1} \in[0,1], \ldots, u_{n} \in[0,1]: \\ u_{1} \nabla \ldots \nabla u_{n}=w}} \mu_{D_{1}}\left(u_{1}\right) \bar{\Delta} \ldots \bar{\Delta} \mu_{D_{n}}\left(u_{n}\right),
$$

где $w \in[0,1]$.

Пусть $R$-нечеткое отношение типа 2 между множествами $X$ и $Y, S$-нечеткое отношение типа 2 между множествами $Y$ и $Z, A$ - НМТ2 с универсальным множеством $X$. Пусть $*$ - некоторая расширенная $t$-норма, $*$ - некоторая расширенная $t$-конорма.

Пусть $Y$ - конечное множество.

Определение 16. Комбинацией нечетких отношений $R$ и $S$ типа 2 называется нечеткое отношение типа 2 между множествами $X$ и $Z$, обозначаемое $R \circ S$, вторичные функции принадлежности которого имеют вид

$$
\mu_{R \circ S}(x, z)=\underset{y \in Y}{\nabla}\left(\mu_{R}(x, y) * \Delta^{*} \mu_{S}(y, z)\right), \quad x \in X, \quad z \in Z .
$$

Пусть $X$ - конечное множество; $Y$ может быть произвольным множеством.

Определение 17. Комбинацией НМТ2 $A$ и нечеткого отношения $R$ типа 2 называется НМТ2 с универсальным множеством $Y$, обозначаемое $A \circ R$, вторичные функции принадлежности которого имеют вид

$$
\mu_{A \circ R}(y)=\underset{x \in X}{\stackrel{\nabla}{*}}\left(\mu_{A}(x) *{ }^{*} \mu_{R}(x, y)\right), \quad y \in Y .
$$

Подчеркнем, что в определениях 16, 17 аргументом всех функций принадлежности является $w$.

Пусть $A_{p}$ - НМТ2 с универсальным множеством $X_{p}, p=1, \ldots, n$. Вторичные функции принадлежности НМТ2 $A_{j}$ будем обозначать $\mu_{A_{j}}\left(x_{j}\right)$, где $x_{j} \in X_{j}$. Каждая из функций $\mu_{A_{j}}\left(x_{j}\right)$ является функцией аргумента $w, w \in[0,1]$.

Определение 18. Декартовым произведением НМТ2 $A_{1}, \ldots, A_{n}$ называется НMT2, обозначаемое $A_{1} \times \cdots \times A_{n}$, универсальным множеством которого является $X_{1} \times \cdots \times X_{n}$, а вторичные функции принадлежности имеют вид

$$
\mu_{A_{1} \times \cdots \times A_{n}}\left(x_{1}, \ldots, x_{n}\right)=\mu_{A_{1}}\left(x_{1}\right) * \stackrel{\Delta}{*} * \mu_{A_{n}}\left(x_{n}\right),
$$

где $*$ - некоторая расширенная $t$-норма. 
Реляционные нечеткие системы называются также нечеткими системами Мамдани. Рассмотрим систему, для которой входом являются действительные числа $x_{1}^{0}, \ldots, x_{m}^{0}$, а выходом - действительное число $y^{0}$. Пусть $x_{1}^{0} \in X_{1}, \ldots, x_{n}^{0} \in X_{n}, y^{0} \in Y ; X_{1}, \ldots, X_{n}, Y$ - некоторые подмножества множества действительных чисел $\mathbb{R}$.

База состоит из $k$ нечетких правил следующего вида:

$$
\text { ЕСЛИ }\left(x_{1}=A_{j 1}\right) \text { И .. И }\left(x_{n}=A_{j n}\right), \text { ТО }\left(y=B_{j}\right),
$$

где $j=1, \ldots, k$. При каждом $j$ считается, что $A_{j p}$ - НMT2 с универсальным множеством $X_{p}$, $p=1, \ldots, n, B_{j}-\mathrm{HMT} 2$ с универсальным множеством $Y$.

Описание нечетких систем Мамдани для случая, когда $A_{j 1}, \ldots, A_{j n}, B_{j}-\mathrm{HMT} 1$, можно найти, например, в [3].

Известными считаются нечеткие отношения $R_{j}$ типа 2 между множествами $X=X_{1} \times \cdots \times X_{n}$ и $Y, j=1, \ldots, k$.

На этапе фазификации по входным данным $x_{1}^{0} \in X_{1}, \ldots, x_{n}^{0} \in X_{n}$ строится НMT2 $A^{\prime}$ с универсальным множеством $X$. На этапе выработки решения сначала находятся НMT2 $B_{j}^{\prime}=A^{\prime} \circ R_{j}$, $j=1, \ldots, k$, а затем с использованием некоторой расширенной $t$-конормы определяется НМТ2 $B^{\prime}$ с вторичными функциями принадлежности

$$
\mu_{B^{\prime}}(y)=\mu_{B_{1}^{\prime}}(y) \stackrel{\nabla}{\nabla} \underset{\nabla}{\nabla} \mu_{B_{n}^{\prime}}(y), \quad y \in Y .
$$

На последнем этапе проводится сначала понижение порядка НMT2 $B^{\prime}$ (например, путем построения центроида $\left.C_{B^{\prime}}\right)$, а затем - дефазификация.

\section{СПИСОК ЛИТЕРАТУРЫ}

1. Лю Б. Теория и практика неопределенного программирования. - М.: БИНОМ. Лаборатория знаний, 2005 .

2. Олизаренко С. А., Перепелица А. В., Капранов В. А. Нечеткие логические системы интервального типа 2. Архитектура и механизм вывода// Сист. обробки інформ. - 2011. - № 5. - С. 156-164.

3. Пегат A. Нечеткое моделирование и управление. - М.: БИНОМ. Лаборатория знаний, 2013.

4. Рутковский Л. Методы и технологии искусственного интеллекта. - М.: Горячая линия - Телеком, 2010.

5. Шведов A. С. Нечеткое математическое программирование: краткий обзор// Пробл. управл. - 2017. - № 3. - C. $2-10$.

6. Шведов А. С. Аппроксимация функций с помощью нейронных сетей и нечетких систем// Пробл. управл. - 2018. - № 1. - С. 21-29.

7. Castillo O., Melin P. Type-2 Fuzzy Logic: Theory and Applications. - Berlin: Springer-Verlag, 2008.

8. Celik E., Gul M., Aydin N., Gumus A. T., Guneri A. F. A comprehensive review of multi criteria decision making approaches based on interval type-2 fuzzy sets// Knowledge-Based Syst. — 2015. — 85. — P. $329-341$.

9. Chang P.-C., Liu C.-H. A TSK type fuzzy rule based system for stock price prediction// Expert Syst. Appl. - 2008. - 34. - P. 135-144.

10. Chang P.-C., Wu J.-L., Lin J.-J. A Takagi-Sugeno fuzzy model combined with a support vector regression for stock trading forecasting// Appl. Soft Comput. - 2016. — 38. — P. 831-842.

11. Du Z., Yan Z., Zhao Z. Interval type-2 fuzzy tracking control for nonlinear systems via sampled-data controller// Fuzzy Sets Syst. - 2019. - 356. - P. 92-112.

12. Hagras H. A hierarchical type-2 fuzzy logic control architecture for autonomous mobile robots// IEEE Trans. Fuzzy Syst. - 2004. - 12. - P. 524-539.

13. Hamza M. F., Yap H. J., Choudhury I. A., Chiroma H., Kumbasar T. A survey on advancement of hybrid type 2 fuzzy sliding mode control// Neural Comput. Appl. — 2018. — 30, № 2. — P. 331-353.

14. Hu B. Q., Wang C. Y. On type-2 fuzzy relations and interval-valued type-2 fuzzy sets// Fuzzy Sets Syst. - 2014. - 236. - P. 1-32.

15. John R. I., Coupland S. Type-2 fuzzy logic: A historical view// IEEE Comput. Intel. Mag. - 2007. — 2, № 1. - P. $57-62$.

16. Karnik N. N., Mendel J. M. Centroid of a type-2 fuzzy set// Inform. Sci. - 2001. - 132. - P. 195-220. 
17. Kumar A., Sharma S., Mitra R. Design of type-2 fuzzy controller based on LQR mapped fusion function// Int. J. Intel. Syst. Appl. - 2012. - 8. - P. 18-29.

18. Mendel J. M. Type-2 fuzzy sets and systems: An overview// IEEE Comput. Intel. Mag. — 2007. — 2, № 1 . - P. 20-29.

19. Mendel J. M. Type-2 fuzzy sets as well as computing with words// IEEE Comput. Intel. Mag. — 2019. — 2, № 1. - P. 82-95.

20. Mendel J. M., Hagras H., Tan W.-W., Melek W. W., Ying H. Introduction to type-2 fuzzy logic control. Theory and applications. - Hoboken (New Jersey): Wiley, 2014.

21. Mizumoto M., Tanaka K. Some properties of fuzzy sets of type 2// Inform. Control. — 1976. — 31. — P. $312-340$.

22. Nieminen J. On the algebraic structure of fuzzy sets of type 2// Kybernetika. — 1977. — 13, № 4. P. 261-273.

23. Tai K., El-Sayed A.-R., Biglarbegian M., Gonzalez C. I., Castillo O., Mahmud S. Review of recent type-2 fuzzy controller applications// Algorithms. - 2016. - 9. - P. 1-19.

24. Tang X., Deng L., Yu J. Qu H. Output feedback predictive control of interval type-2 T-S fuzzy systems with markovian packet losses// IEEE Trans. Fuzzy Syst. — 2018. — 26. — P. 2450-2459.

25. Torshizi A. D., Zarandi M. H. F., Zakeri H. On type-reduction of type-2 fuzzy sets: A review// Appl. Soft Comput. - 2015. - 27. - P. 614-627.

26. Wagner C., Hagras H. Toward general type-2 fuzzy logic systems based on zSlices// IEEE Trans. Fuzzy Syst. - 2010. - 18, № 4. - P. 637-660.

27. Xiao B., Lam H. K., Yang X., Yu Y., Reu H. Tracking control design of interval type-2 polynomial-fuzzymodel-based systems with time-varying delay// Engrg. Appl. Art. Intel. — 2018. — 75. — P. 76-87.

28. Zadeh $L$. A. The concept of a linguistic variable and its application to approximate reasoning, I// Inform. Sci. - 1975. - 8. - P. 199-249.

29. Zhang Z., Niu Y. Adaptive sliding mode control for interval type-2 stochastic fuzzy systems subject to actuator failures// Int. J. Syst. Sci. - 2018. - 49. - P. 3169-3181.

30. Zhou H.-B., Garibaldi J. M., John R. I., Chiclana F. On constructing parsimonious type-2 fuzzy logic systems via influential rule selection// IEEE Trans. Fuzzy Syst. — 2009. — 17, № 4. — P. 654-667.

Шведов Алексей Сергеевич

Национальный исследовательский университет «Высшая школа экономики», Москва

E-mail: ashvedov@hse.ru 